\title{
Muscle activation during body weight-supported locomotion while using the ZeroG
}

\author{
Alyssa M. Fenuta, HBSc; Audrey L. Hicks, PhD* \\ Department of Kinesiology, McMaster University, Hamilton, ON, Canada
}

\begin{abstract}
The ZeroG provides dynamic body weight support (BWS) using a harness while individuals with mobility impairments (e.g., spinal cord injury) ambulate overground. Muscle activity during locomotion using this device was studied in 13 nondisabled adults (age $23.8+/-2.7$ yr). Electromyography (EMG) recordings were collected from tibialis anterior (TA), medial gastrocnemius (MG), rectus femoris (RF), and biceps femoris muscles during randomized walking trials at preferred speeds under five levels of BWS (0\%, 20\%, 40\%, 60\%, 80\%). Filtered EMG signals from each trial were normalized to $0 \%$ BWS and correlated with gait phases. Muscle activity, averaged across muscles, decreased significantly at heel strike by $33.4 \%$ with increasing BWS. Offloading significantly decreased heel strike activity of RF (62.8\%), MG (35.5\%), and TA (25.9\%). Gait cycle completion time increased with BWS primarily because of increased swing phase time. These results summarizing the effect of BWS on muscle activation during ambulation can now be compared with clinical populations using the ZeroG.
\end{abstract}

Key words: dynamic body weight support, electromyography, gait, locomotor training, mobility impairments, muscle activity, overground walking, rehabilitation, spinal cord injury, ZeroG.

\section{INTRODUCTION}

Body weight-supported locomotor training has created enthusiasm in clinical populations and service providers as a rehabilitation tool to improve overground walking ability and other health outcomes [1]. Varying the amount of body weight supported by such devices alters the intensity of training sessions; unloading the individual decreases the muscular demands of the lower limbs [2]. For individuals with neurological impairments (e.g., spinal cord injury) evidence suggests that by offsetting body weight, appropriate gait pattern expression may be facilitated and even normalized. Adult spinal cat models provided initial indications of the possibility of locomotor recovery following interactive treadmill gait training programs, which provided graded body weight support (BWS) [3]. Persons with spinal cord injury (complete and incomplete) have demonstrated the ability to increase weight-bearing capacity of lower-limb extensors through body weight-supported treadmill training, in addition to increasing appropriate gastrocnemius and decreasing inappropriate tibialis anterior (TA) activation [4]. An overground body weight-supported walking study (0/30/100\% BWS at natural cadence) in individuals with chronic stroke found offloading resulted in reduced hip range of motion, with individuals responding to the support by decreasing their walking speed and taking shorter strides [5].

As this information suggests, there are a variety of therapeutic training approaches that offset body weight

\footnotetext{
Abbreviations: $\mathrm{BF}=$ biceps femoris, $\mathrm{BWS}=$ body weight support, $\mathrm{EMG}=$ electromyography, $\mathrm{MG}=$ medial gastrocnemius, $\mathrm{RF}=$ rectus femoris, $\mathrm{TA}=$ tibialis anterior.

*Address all correspondence to Audrey L. Hicks, PhD; Department of Kinesiology, McMaster University, 1280 Main St West, Hamilton, ON, Canada L8S 4L8; 905-525-9140, ext 24643; fax: 905-523-6011. Email: hicksal@mcmaster.ca http://dx.doi.org/10.1682/JRRD.2013.01.0005
} 
as a patient progresses through his or her rehabilitation program. These options include manual or roboticassisted treadmill training, as well as treadmill or overground training with or without electrical stimulation of the peroneal nerve [6]. Each training approach has its respective advantages and disadvantages; for example, robotic-assisted training provides stepping consistency but does not allow the movement variability associated with overground walking [7]. In addition, this therapeutic option is treadmill-based and, as a result, is not as taskspecific or transferable to daily living as overground training programs [5].

Currently, there is a need for a body weight-supported overground walking device equipped with a motor that moves along an overhead track independent of the user in order to eliminate the possibility that a drag force created by the system itself affects gait performance [5]. The newly developed ZeroG system (Aretech LLC; Ashburn, Virginia), created by Hidler et al., attempts to provide this dynamic BWS through the use of a custom-series elastic actuator during overground walking and balance training (Figure 1) [8]. It has been suggested that more natural ground reaction forces and gait characteristics can be achieved through the use of dynamic BWS [8]. To date, no study has investigated the effects of BWS on muscle activation patterns during walking using the ZeroG. It would be beneficial to know "normal" responses to partial weight bearing using this device before using it in a rehabilitation setting. Therefore, the purpose of this study was to collect normative data from walking trials in nondisabled participants using the ZeroG at varying degrees of BWS (0/20/40/60/80\% BWS). We hypothesized that decreased levels of activation primarily in the ankle plantar flexors (e.g., calves) would be experienced with increased BWS. The results from this study will eventually be used as a model for comparison with individuals that have mobility impairments who use this rehabilitation tool for gait therapy.

\section{METHODS}

\section{Subjects}

Thirteen nondisabled individuals (6 males, 7 females) with an average age of $23.8 \pm 2.7 \mathrm{yr}$ and weight of $68.3 \pm$ $8.0 \mathrm{~kg}$ participated in the study. "Nondisabled" was defined as an individual without musculoskeletal disease (e.g., multiple sclerosis, cerebral palsy) or injury within

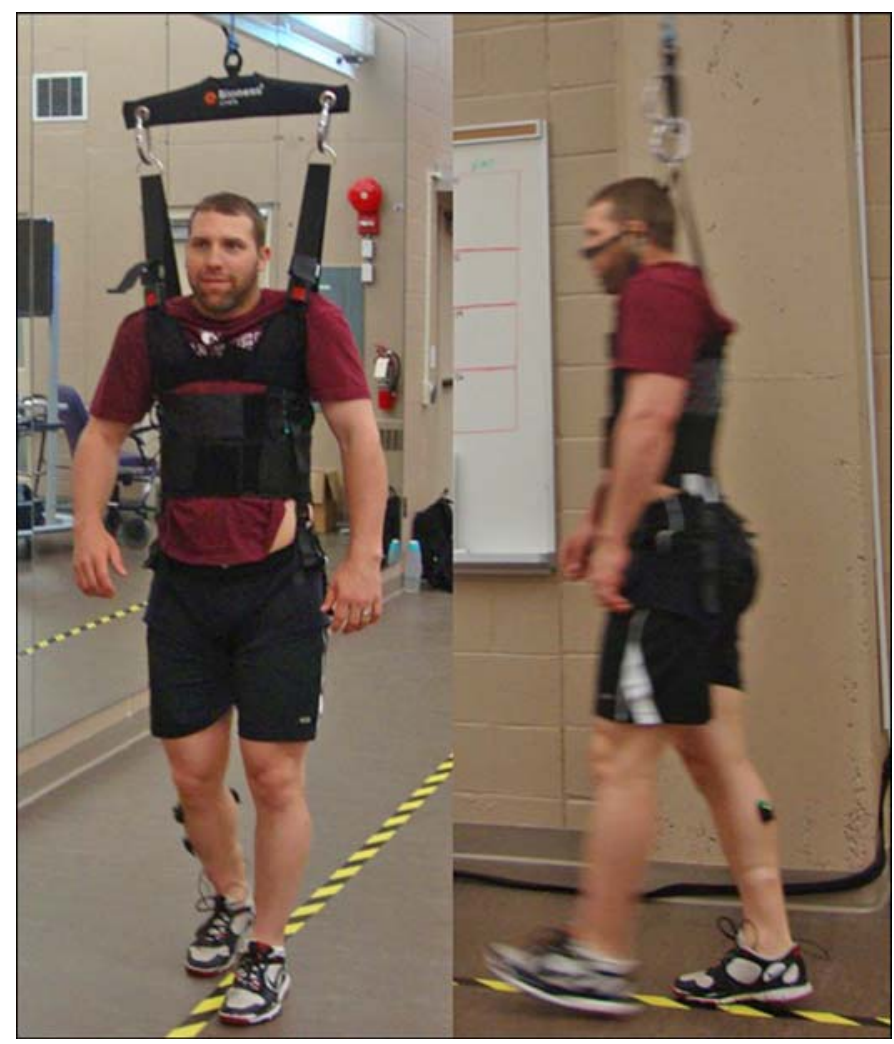

Figure 1.

ZeroG overground body weight support (BWS) system. Custom-series elastic actuator travels along overhead trolley providing static and dynamic BWS, allowing individuals to safely practice activities of daily living.

the past year (e.g., sprain, strain) of the lower leg and who was able to comfortably walk 30 m unassisted.

\section{Protocol}

Subjects were informed prior to testing to wear comfortable clothing including a T-shirt, shorts, socks, and running shoes. All testing took place at the Robert Fitzhenry Specialized Rehabilitation and Exercise Laboratory in the Ivor Wynne Centre at McMaster University (Hamilton, Ontario) in accordance with the approved protocol by the McMaster Research Ethics Board.

Delsys Trigno wireless electromyography (EMG) surface electrodes (Delsys Inc; Boston, Massachusetts) were attached to the TA, rectus femoris (RF), medial gastrocnemius (MG), and biceps femoris (BF) muscles of the right leg. A Delsys wireless force-sensitive resistor (footswitch) was attached to the right foot with sensors placed on the base of the great toe, first and fifth metatarsal 
heads, and heel. Body weight was taken using an electronic scale after electrode and footswitch attachment was complete and with subjects wearing their shoes during the measurement. Finally, participants were fitted to the appropriate size ZeroG harness and were given the opportunity to practice walking at all of the various levels of BWS (0/20/40/60/80\%); the ZeroG BWS system has been described in detail previously [8].

The EMGworks Acquisition software program (Delsys EMGworks 4.0) was used to collect muscle activity data from the randomized BWS trials. All participants initially completed baseline trials while walking at natural cadence along a $15 \mathrm{~m}$ track at 0 percent BWS. BWS in subsequent trials was randomized between four different levels (20/40/60/80\%); 3 trials were performed at each level for a total of 15 trials, all at the subject's respective natural cadence.

\section{Statistical Analysis}

EMGworks Analysis and Statistica 8 (StatSoft; Tulsa, Oklahoma) software programs were used for analysis of acquired data; trials for each level were kept separate. To investigate change in EMG activity at different levels of BWS, the root mean square of the filtered signal energy was determined over three gait cycles and normalized to the average signal at baseline (0\% BWS). A similar approach was used to assess changes in gait cycle components (e.g., swing and stance phase) with increasing support by using the footswitch signal to determine when the foot made contact with the ground. The effects of BWS on muscle activation and gait cycle components were analyzed with repeated measure analysis of variance using the filtered EMG signals. Tukey honest significant difference was used for post hoc testing when necessary. Statistical significance was set at $p<0.05$ for all analyses.

\section{RESULTS}

No significant differences were found between trials or sexes; therefore, the described results represent the combined data from the second trial.

\section{Muscle Activity During Gait Cycle (Heel Strike Versus Toe-Off)}

The EMG profiles of muscle groups were nearly identical despite variations in the amount of body weight offset; however, significant differences were observed with respect to amplitude (Figure 2). Averaged muscle activity across the gait cycle decreased significantly at $\geq 40$ percent $B W S$, with a mean maximum reduction of 23.7 percent at 80 percent BWS compared with baseline (Figure 3). This difference was attributed to a decrease in heel strike muscular activity as BWS increased, with a mean maximum reduction of 33.4 percent at 80 percent BWS compared with baseline; no significant differences were found in muscle activity during toe-off with increasing levels of BWS. Looking at the individual muscle groups, MG activity significantly decreased at $\geq 40$ percent BWS, with RF and TA demonstrating significant decreases at higher levels of BWS (60\%-80\% BWS). The decline in BF activity with increasing levels of BWS was not significantly different from baseline. Although MG activity was the first muscle group at heel strike to be affected by increasing levels of BWS, RF was most affected by BWS, with a 62.8 percent decrease in activity at the highest level of BWS (80\%) relative to baseline (Figure 4).

\section{Time to Complete Gait Cycle (Stance Versus Swing Phase)}

The gait cycle took longer to complete with greater BWS, increasing by 25.8 percent at the highest level of BWS (Figure 5). Swing and stance phase times were both significantly longer at $\geq 40$ percent BWS, but the relative change was greater in the swing phase time $(46.5 \%$ vs $13.8 \%$, respectively).

\section{DISCUSSION}

To our knowledge, this is the first study to look at muscle activation patterns during gait with increasing levels of BWS using the ZeroG training system.

\section{Muscle Activity}

Our data agrees with previous descriptions of "normal” patterns of muscle activation during walking with 0 percent BWS [9-10]. Some of these similarities include weight-accepting muscles (TA, RF, BF) peaking in activity during the first 15 percent of stride and distal supporting muscles (TA, MG) tending to be more active than proximal muscles (BF, RF). Differences in muscular activity during the walking trials in the present study were not significantly affected until 40 percent or more body weight was offset by the ZeroG training system. This is consistent with previous research evaluating body 


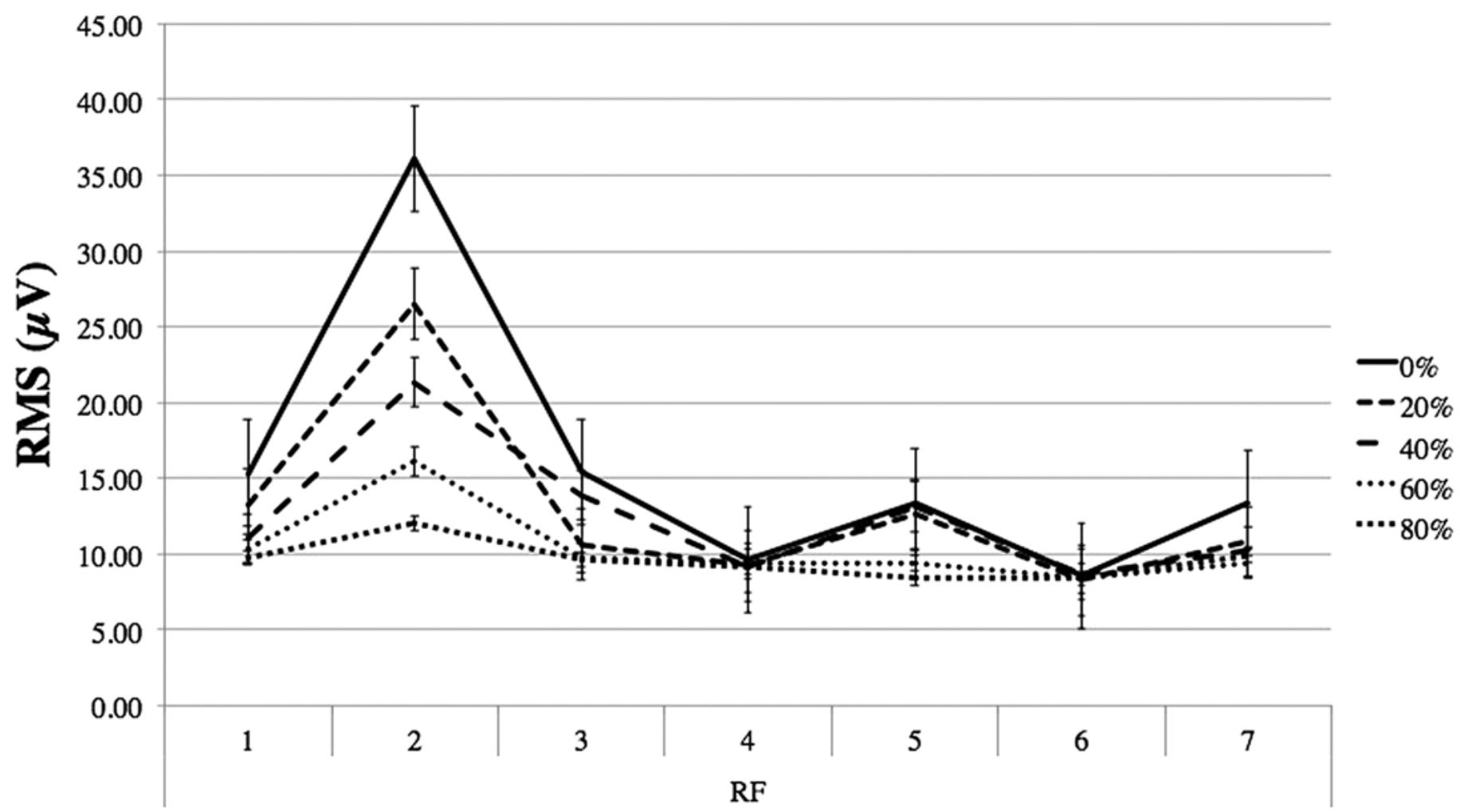

Gait Phase

Figure 2.

Pattern of hip flexor (rectus femoris [RF]) muscle activity during different phases of gait with varying percentage body weight support. Phases of gait were defined as follows: (1) beginning of heel strike, (2) maximum heel strike, (3) cross between heel strike and toe-off (foot flat), (4) maximum toe-off, (5) end toe-off, (6) midswing, and (7) end of cycle. Values are mean \pm standard error. RMS = root mean square.

weight-supported training, which demonstrated that gait kinematics similar to baseline walking could be maintained when 30 percent or less support was provided [2].

In agreement with Hidler et al. [8], increasing BWS decreased muscular demand of the lower limbs during the walking trials, thereby decreasing the intensity of the task. In the present study, lower-limb hip flexors and ankle plantarflexors were more affected by BWS (RF > MG) than ankle dorsiflexors and hip extensors (TA > $\mathrm{BF}$ ). We hypothesized that the plantar flexor muscle group would be most affected by increasing levels of BWS; although MG activity was significantly affected at a lower level of offloading ( $40 \%$ BWS), the greatest decline in muscle activity with increasing BWS occurred in RF. RF activity was most affected at heel strike, and its activity decreased by as much as 63 percent at the highest level of BWS. Interestingly, at higher levels of BWS, RF's second activity burst, which is associated with for- ward acceleration of the limb in early swing, disappeared. Yang and Winter have attributed this disappearance to slower walking cadences for which the acceleration required to swing the limb is low enough that the initial part of swing can be accomplished with minimal muscle activity, similar to a pendulum [10].

In this study, the nonsignificant change in BF activity may have been a result of large between-subject variability at the highest level of BWS, possibly because of subjects resisting the vertical support from the ZeroG training system in an attempt to maintain "normal" gait kinematics. The decrease in natural cadence at the highest levels of BWS also may have contributed to the increased variability in the signaling, a relationship highlighted in a study by van Hedel et al. [11]. The aforementioned study involved nondisabled subjects performing body weightsupported treadmill walking at 0/25/50/75 percent BWS. Similar to the results in our study, the EMG patterns 


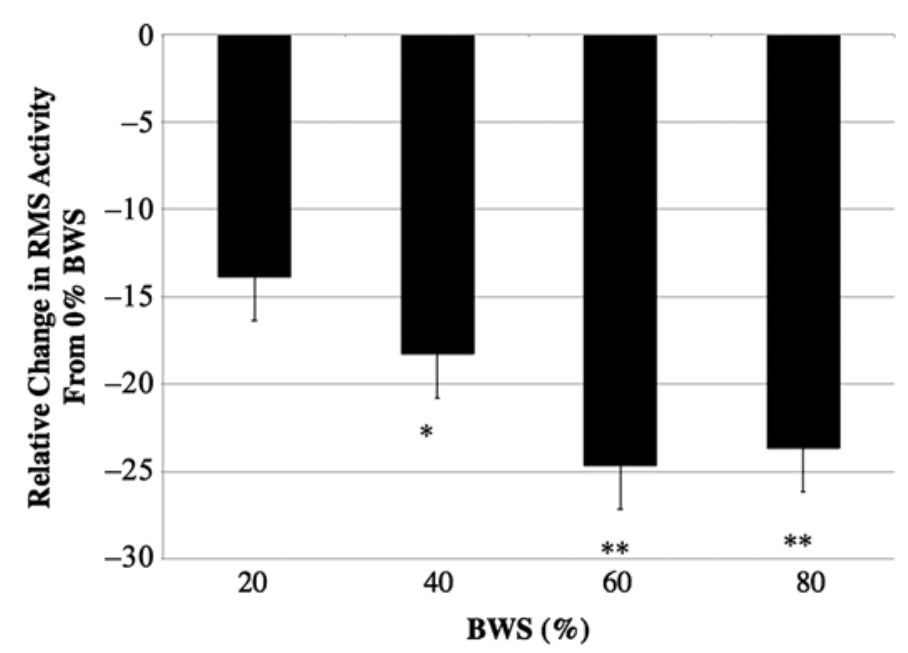

Figure 3.

Overall muscle activity normalized to 0 percent body weight support (BWS). Values are mean \pm standard error. ${ }^{\star} p<0.05,{ }^{\star \star} p<$ $0.01 . \mathrm{RMS}=$ root mean square.

indicated RF and MG activity to be most influenced at the highest level of BWS, with only slight differences detected in TA activity. In contrast to our data, van Hedel et al. found large differences in lateral hamstring activation. Comparison of this data with the present study highlights the important differences that need to be considered when comparing overground and treadmill walking (e.g., hip range of motion) and suggests that knee kinematics may be more comparable than hip kinematics between the two modalities.

\section{Gait Cycle Time}

The slower natural cadence of participants in this study at $\geq 40$ percent BWS supports previous research indicating that at higher levels of BWS individuals have difficulty moving their center of mass over their base of support [6]. It is also likely that subjects experienced reduced acceleration rates during locomotion at higher levels of BWS, which decreased walking velocity and step length, contributing to the prolonged gait cycle.

In the present study, increases in both swing and stance (ground contact) phase contributed to the slower gait cycle, with a greater contribution attributed to increased swing phase completion time by as much as 46.5 percent. This indicates that, at higher levels of BWS, individuals spend significantly more time balancing a portion of their body weight on one limb, while the opposite limb completes swing phase. This is in contrast to a previous overground BWS training study in persons with stroke, in which no changes in stance or swing period duration were evident with increasing BWS [5]. These differences may be attributed to the clinical population, who potentially responded differently to the BWS, in addition to the different overground training devices used; the study by Sousa et al. used a device with an electric motor that relied on the individual for movement [5] versus the current study in which the ZeroG uses a custom-series elastic actuator that moves independently of the participant.

\section{Limitations}

The fact that walking cadence was not controlled in the present study could be considered a limitation; however, all participants received the same instructions to walk at their natural cadence during the different trials. The sensitivity of EMG profiles to walking velocity is well known, resulting in decreased amplitude and increased variability of muscle activity at slower cadences. Changes in walking cadence affect the acceleration of the lower limb, which, according to Winter, affects hip and knee musculature activity more than the ankle [12]. Future studies using the ZeroG that impose restrictions to step length (e.g., using floor tape markers) and walking velocity (e.g., using a metronome) would provide important information into the potential interaction effect of these two factors with changing levels of BWS while using this device. These investigations will also provide a means of comparison to other studies, including supported treadmill-based walking studies, which have the ability to control for speed in addition to BWS. The clinical significance of the small differences observed in cadence and stride length still need to be determined. In addition, the difference between overground and treadmill walking needs to be acknowledged, because the treadmill belt, through its facilitation of hip extension, may alter the effect of offloading body weight compared with an overground device. The importance of these comparisons will better inform therapists in terms of modality selection, which may be modified based on the goal of the rehabilitation program for an individual.

The purpose of this study was to establish normative data (using nondisabled participants) describing the effect of BWS on muscle activation utilizing the ZeroG overground training device. The potential generalizability of the presented results in clinical populations with mobility impairments is questionable given the unique and wide-ranging functional limitations associated with 


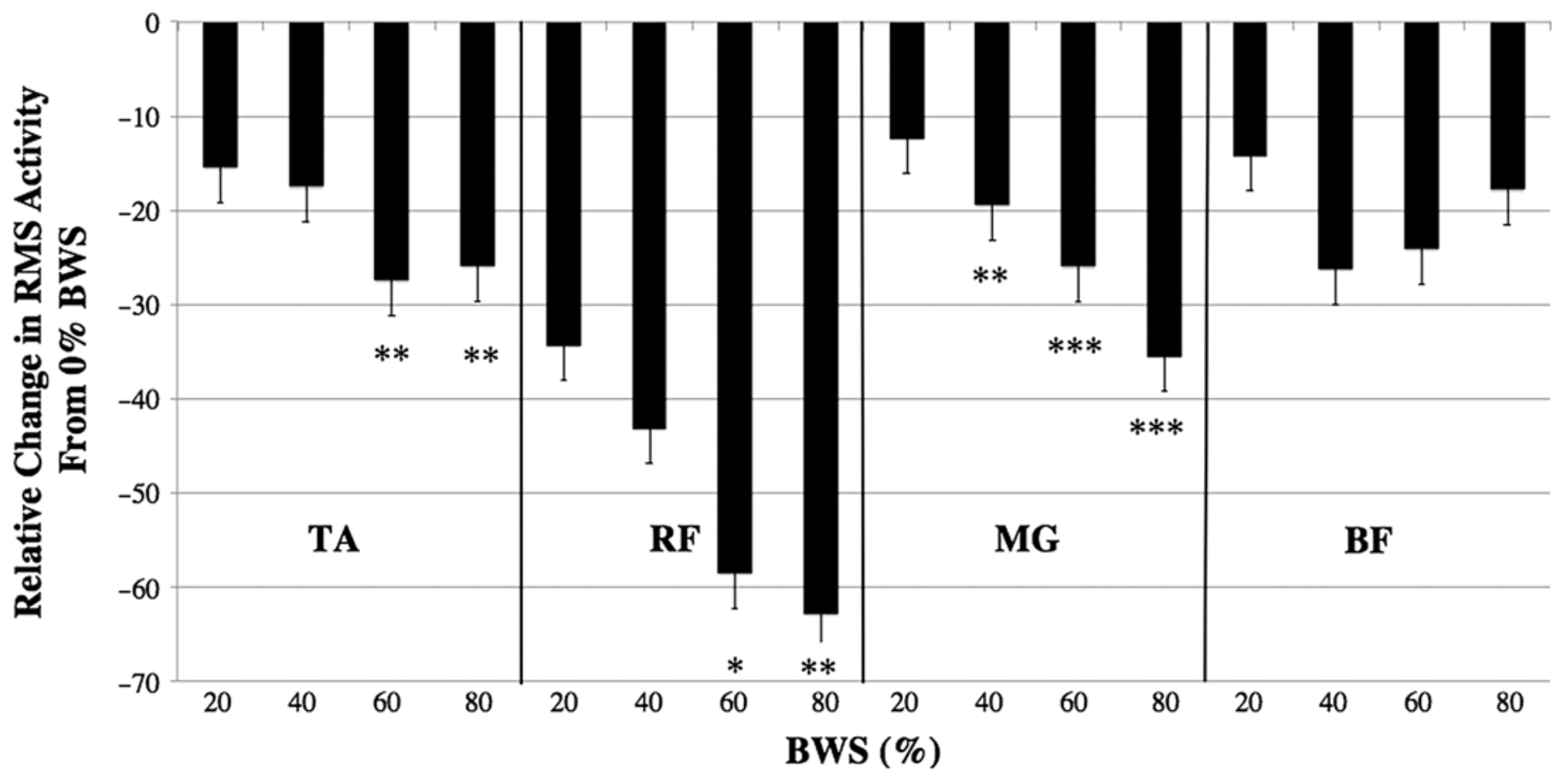

Figure 4.

Heel strike muscle activity normalized to 0 percent body weight support (BWS). Root mean square (RMS) of tibialis anterior (TA), rectus femoris (RF), medial gastrocnemius (MG), and biceps femoris (BF). Values are mean \pm standard error. ${ }^{*} p<0.05,{ }^{\star \star} p<0.01,{ }^{\star \star *} p<0.001$.

impairments affecting gait. For example, it has been found that patients with neurological impairment lack an adequate push-off secondary to abnormal muscle activation during gait [2]. The important next step is to repeat this study using clinical populations in order to better understand the neuromuscular adaptations to BWS while using the ZeroG device.

\section{CONCLUSIONS}

This is the first study to look at changes in gait patterns with increasing levels of BWS using the ZeroG training system. The EMG profiles of muscle groups in this study were nearly identical despite variations in the amount of body weight offset; however, significant differences were observed with respect to amplitude. Thus, it can be concluded that the dynamic BWS provided by the ZeroG decreases the muscular demand of the lower limbs without significantly altering muscle activation patterns during gait. Future research in clinical populations with mobility impairments (e.g., spinal cord injury, stroke) using this device should be conducted in order to compare the muscular activation response with the data acquired in the present study. This will help in the development of more effective and functional rehabilitation programs for these populations. The evidence from this study also emphasizes the importance of incorporating

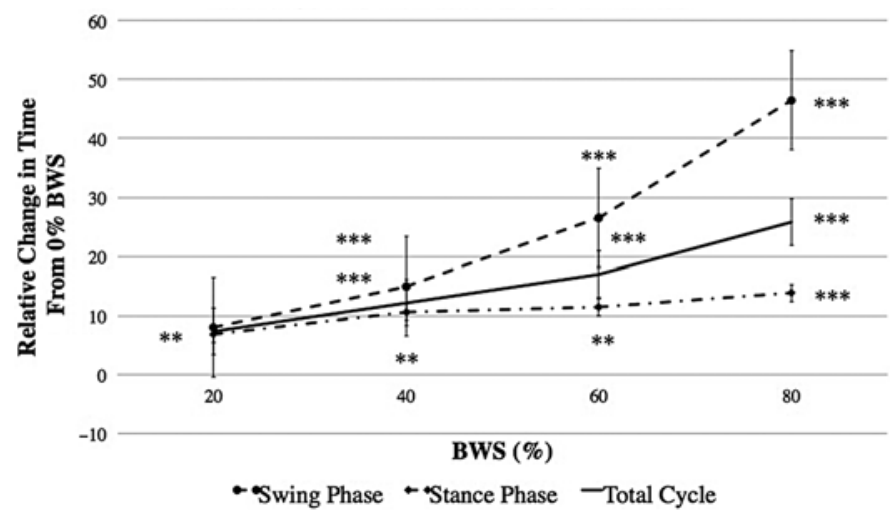

Figure 5.

Time to complete gait cycle normalized to 0 percent body weight support (BWS). Values are mean \pm standard error. ${ }^{* \star} p<$ $0.01,{ }^{* \star *} p<0.001$. 
balance exercises into gait therapy as overground walking with $\geq 40$ percent BWS forces individuals to support a portion of their body weight on one limb for a significantly longer period of time.

\section{ACKNOWLEDGMENTS}

\author{
Author Contributions: \\ Study concept and design: A. M. Fenuta, A. L. Hicks. \\ Acquisition of data: A. M. Fenuta. \\ Analysis and interpretation of data: A. M. Fenuta. \\ Drafting of manuscript: A. M. Fenuta. \\ Critical revision of manuscript for important intellectual content: \\ A. L. Hicks. \\ Study supervision: A. L. Hicks.
}

Financial Disclosures: The authors have declared that no competing interests exist.

Funding/Support: This work was unfunded th the time of manuscript preparation.

Additional Contributions: Alyssa M. Fenuta completed this study during her Master's studies in the Department of Kinesiology at McMaster University, Hamilton, Ontario, Canada. Having completed her Master's degree, she is now affiliated with the School of Kinesiology and Health Studies, Queen's University, Kingston, Ontario, Canada.

Institutional Review: This study received approval from the McMaster Research Ethics Board (Hamilton, Ontario, Canada).

Participant Follow-Up: The authors do not plan to inform participants of the publication of this study.

\section{REFERENCES}

1. Hicks AL, Ginis KA. Treadmill training after spinal cord injury: It's not just about the walking. J Rehabil Res Dev. 2008;45(2):241-48. [PMID:18566942] http://dx.doi.org/10.1682/JRRD.2007.02.0022

2. Finch L, Barbeau H, Arsenault B. Influence of body weight support on normal human gait: Development of a gait retraining strategy. Phys Ther. 1991;71(11):842-55, discussion 855-56. [PMID:1946621]

3. Barbeau H, Rossignol S. Recovery of locomotion after chronic spinalization in the adult cat. Brain Res. 1987;412(1):84-95. [PMID:3607464] http://dx.doi.org/10.1016/0006-8993(87)91442-9

4. Dietz V. Body weight supported gait training: From laboratory to clinical setting. Brain Res Bull. 2008;76(5):459-63. [PMID:18534251] http://dx.doi.org/10.1016/j.brainresbull.2008.02.034
5. Sousa CO, Barela JA, Prado-Medeiros CL, Salvini TF, Barela AM. The use of body weight support on ground level: An alternative strategy for gait training of individuals with stroke. J Neuroeng Rehabil. 2009;6:43.

[PMID:19951435] http://dx.doi.org/10.1186/1743-0003-6-43

6. Field-Fote EC, Lindley SD, Sherman AL. Locomotor training approaches for individuals with spinal cord injury: A preliminary report of walking-related outcomes. J Neurol Phys Ther. 2005;29(3):127-37. [PMID:16398945] http://dx.doi.org/10.1097/01.NPT.0000282245.31158.09

7. Hidler J, Wisman W, Neckel N. Kinematic trajectories while walking within the Lokomat robotic gait-orthosis. Clin Biomech (Bristol, Avon). 2008;23(10):1251-59. [PMID:18849098] http://dx.doi.org/10.1016/j.clinbiomech.2008.08.004

8. Hidler J, Brennan D, Black I, Nichols D, Brady K, Nef T. ZeroG: Overground gait and balance training system. J Rehabil Res Dev. 2011;48(4):287-98. [PMID:21674384] http://dx.doi.org/10.1682/JRRD.2010.05.0098

9. Winter D. The biomechanics and motor control of human gait: Normal, elderly and pathological. Waterloo (Canada): University of Waterloo Press; 1987. p. 47-80.

10. Yang JF, Winter DA. Surface EMG profiles during different walking cadences in humans. Electroencephalogr Clin Neurophysiol. 1985;60(6):485-91. [PMID:2408847] http://dx.doi.org/10.1016/0013-4694(85)91108-3

11. van Hedel HJ, Tomatis L, Müller R. Modulation of leg muscle activity and gait kinematics by walking speed and bodyweight unloading. Gait Posture. 2006;24(1):35-45. [PMID:16099161] http://dx.doi.org/10.1016/j.gaitpost.2005.06.015

12. Winter DA. Biomechanical motor patterns in normal walking. J Mot Behav. 1983;15(4):302-30. [PMID:15151864] http://dx.doi.org/10.1080/00222895.1983.10735302

Submitted for publication January 8, 2013. Accepted in revised form July 25, 2013.

This article and any supplementary material should be cited as follows:

Fenuta AM, Hicks AL. Muscle activation during body weight-supported locomotion while using the ZeroG. J Rehabil Res Dev. 2014;51(1):51-58. http://dx.doi.org/10.1682/JRRD.2013.01.0005

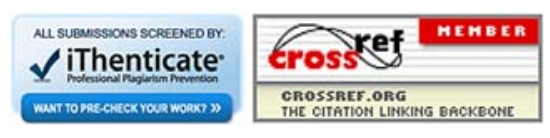


